\title{
EFECTO DEL ALMACENAMIENTO SOBRE, UCHUVA ADICIONADA CON COMPONENTES FISIOLÓGICAMENTE ACTIVOS Y DESHIDRATADA POR AIRE CALIENTE
}

\section{EFFECT OF STORAGE OF HOT DRIED CAPE GOOSEBERRY ADDED WITH PHYSIOLOGICALLY ACTIVE COMPONENTS BY VACUUM IMPREGANTION}

\author{
Misael Cortés-Rodríguez ${ }^{1}$, Yudy Ana Cabrera Ordoñez ${ }^{2}$, Rodrigo Ortega-Toro ${ }^{3}$
}

\begin{abstract}
${ }^{1}$ Ing. Químico, Ph.D. Ingeniería de Alimentos, Profesor Titular. Universidad Nacional de Colombia sede Medellín, Departamento de Ingeniería Agrícola y de Alimentos. Calle 59A No. 63-120, Medellín, Colombia, e-mail: mcortesro@unal.edu.co, (iDhttps://orcid.org/0000-0003-3407-1635; ${ }^{2}$ Ing. Agroindustrial, M.Sc, Ciencia y Tecnología de Alimentos. Universidad Mariana, Calle 18 No. 34-104, Pasto-Nariño, Colombia, e-mail: ycabrera@umariana.edu.co, ; ${ }^{3}$ Ing. Agroindustrial, M.Sc. Ciencia e Ingeniería de los alimentos, Ph.D. Ciencia, Tecnología y Gestión Alimentaria, Profesor Auxiliar. Universidad de Cartagena, Programa de Ingeniería de Alimentos, Departamento de Ciencias de los Alimentos. Cra. 6 No. 36-100, Cartagena, Colombia, e-mail: rortegap1@unicartagena.edu.co, (Dhttps://orcid.org/0000-0003-0815-5317.
\end{abstract}

Rev. U.D.C.A Act. \& Div. Cient. 21(1): 427-437, Julio-Diciembre, 2018 https://doi.org/10.31910/rudca.v21.n2.2018.989

Artículo de acceso abierto publicado por Revista U.D.C.A Actualidad \& Divulgación Científica bajo una licencia Creative Commons CC BY-NC 4.0

\section{RESUMEN}

El objetivo de la investigación fue evaluar el efecto de la temperatura $\left(4,20\right.$ y $\left.30^{\circ} \mathrm{C}\right)$, tiempo $(0,1,2,3,4,5$ y 6 meses $)$ y las condiciones del empacado (con y sin vacío), durante el almacenamiento de uchuvas adicionadas por impregnación al vacío con calcio y vitaminas $\mathrm{B}_{9}, \mathrm{C}, \mathrm{D}_{3}, \mathrm{E}$ y deshidratadas por aire, a $60^{\circ} \mathrm{C}$ y $2 \mathrm{~m} / \mathrm{s}$. El efecto del empacado no fue un factor crítico en la estabilidad de los componentes fisiológicamente activos (CFA). El calcio fue estable a las condiciones de almacenamiento evaluadas. Las vitaminas $\mathrm{B}_{9}, \mathrm{C}$ y $\mathrm{D}_{3}$ disminuyen con el incremento del tiempo y la temperatura de almacenamiento, mientras que la vitamina $\mathrm{E}$ presenta degradación. Las mejores condiciones de almacenamiento durante los 6 meses fueron $4^{\circ} \mathrm{C}$ y empacado atmosférico, alcanzando valores de 434,0 \pm 45,0mg; 179, $1 \pm 89,2 \mu \mathrm{g} ; 28,3$ $\pm 5,4 \mathrm{mg} ; 3,5 \pm 1,1 \mu \mathrm{g}$ y $8,7 \pm 1,2 \mathrm{mg}$, para Ca y vitaminas $\mathrm{B}_{9}, \mathrm{C}, \mathrm{D}_{3}$ y E, respectivamente, cumpliendo con el descriptor "Excelente fuente", en todos los nutrientes evaluados, en una porción de $100 \mathrm{~g}$, según la normativa colombiana. La técnica de impregnación a vacío es un pretratamiento efectivo para la incorporación de CFA, contribuyendo en la generación de valor de la agrocadena de uchuva.

Palabras clave: alimentos funcionales, conservación de alimentos, deshidratación, vitaminas, minerales. www.decs.bvs.br.

\section{ABSTRACT}

The aim of the research was to evaluate the effect of temperature $\left(4,20\right.$ and $\left.30^{\circ} \mathrm{C}\right)$, time $(0,1,2,3,4,5$ and 6 months) and the packaging conditions (with and without vacuum), during the storage of gooseberries added with calcium and vitamins $B_{9}, C, D_{3}, E$ by means of vacuum impregnation and then dehydrated by air at $60^{\circ} \mathrm{C}$ and $2 \mathrm{~m} / \mathrm{s}$. The effect of the packing was not a critical factor in the stability of the physiologically active components (PAC). The calcium was stable at the storage conditions evaluated. Vitamins $\mathrm{B}_{9}, \mathrm{C}$ and $\mathrm{D}_{3}$ decreased with increasing storage time and temperature; whereas, vitamin E showed degradation. The best storage conditions during the 6 months were $4^{\circ} \mathrm{C}$ and atmospheric packing, reaching values of $434,0 \pm 45,0 \mathrm{mg}$; $179,1 \pm 89,2 \mathrm{mg} ; 28,3 \pm 5,4 \mathrm{mg} ; 3,5 \pm 1,1 \mathrm{~g}$ y $8,7 \pm 1,2 \mathrm{mg}$ for calcium and vitamins $\mathrm{B}_{9}, \mathrm{C}, \mathrm{D}_{3}$ y E respectively, complying with the descriptor "Excellent source" in all evaluated nutrients in a portion of $100 \mathrm{~g}$ according to the Colombian regulations. The technique of vacuum impregnation is an effective pretreatment for the incorporation of PAC, contributing to the generation of value of the cape gooseberry agro-chain.

Keywords: functional foods, food preservation, dehydration, vitamins, minerals. www.decs.bvs.br. 


\section{INTRODUCCIÓN}

La uchuva (Physalis peruviana L.) es una fruta exótica que se cultiva en casi todos los altiplanos de los países tropicales, subtropicales e, incluso, templados, siendo Colombia su principal productor (Álvarez-Herrera et al. 2015). Se le atribuyen propiedades medicinales y se destaca por su alto nivel en ácido ascórbico, además de $\beta$-caroteno, $\mathrm{Fe}, \mathrm{Ca}$, entre otros (Olivares-Tenorio et al. 2017). Este fruto ha sido identificado como fruto exótico entre una serie de productos con potencial agroindustrial, por sus características fisicoquímicas (Carvalho et al. 2015), propiedades nutricionales (Olivares-Tenorio et al. 2017) y medicinales (Rabie et al. 2015), adquiriendo gran importancia por su constante demanda en los mercados europeos y norteamericanos.

Los alimentos funcionales proporcionan un efecto benéfico para la salud sobre una o más funciones en el organismo, más allá de su valor nutricional básico, de forma que permita, tanto mejorar la salud como reducir el riesgo de algunas enfermedades (Bigliardi \& Galati, 2013). Los mismos autores mencionan que estos efectos, se encuentran relacionados con la adición de vitaminas y de minerales, identificados como componentes fisiológicamente activos (CFA). La OMS realizó una selección de CFA, que interactúan con las enfermedades de mayor recurrencia, destacándose el Ca y las vitaminas $\mathrm{B}_{9}, \mathrm{C}, \mathrm{D}_{3}$ y $\mathrm{E}$, cuyas deficiencias se relacionan con problemas de mineralización ósea, síntesis de proteínas, estrés oxidativo, entre otros (WHO, 2003).

Ha sido confirmada la posibilidad de aplicar la técnica de impregnación a vacío (IV), para la incorporación de CFA, en matrices porosa de frutas (Castagnini et al. 2015; Betoret et al. 2015); consiste en el intercambio del gas o del líquido ocluido en los poros o espacios extracelulares, por una disolución externa, mediante la acción de mecanismos hidrodinámicos (HDM), por gradientes de presión y fenómenos de deformación-relajación (FDR) (Carciofi et al. 2015). Los frutos IV, al ser sometidos a procesos de conservación, como el secado por aire caliente (SAC), remueven, parcialmente, su contenido de agua, permitiendo ampliar su vida útil al disminuir su actividad del agua (aw) (Tappi et al. 2016); así, se disminuye la disponibilidad y la movilidad de agua en los alimentos y se reduce la posibilidad de reacciones químicas, bioquímicas y de crecimiento de microorganismos que produzcan alteraciones (Stevenson et al. 2015).

El objetivo del trabajo fue evaluar el efecto de la temperatura, el tiempo y las condiciones de empacado en uchuvas adicionadas por IV con calcio y vitaminas $\mathrm{B}_{9}, \mathrm{C}, \mathrm{D}_{3}$, E y deshidratadas por aire, durante el almacenamiento.

\section{MATERIALES Y MÉTODOS}

Materias primas: Se utilizaron uchuvas frescas (UF) (Ecotipo Colombia), con peso entre $5 \mathrm{y} 7 \mathrm{~g}$ y con estado de madurez entre 3 y 5, según la Norma Técnica Colombiana 4580 de 2004. Se utilizó una emulsión de impregnación (EI), cuya formulación contenía cloruro y fumarato de calcio, como fuentes de $\mathrm{Ca}$, vitaminas $\mathrm{B}_{9}$ (ácido fólico), $\mathrm{C}, \mathrm{D}_{3}$ (colecalciferol) y E (DL- $\alpha$-tocoferol acetato), proteína aislada de soja, sucralosa, Tween 80 y Span 60, grado alimentario.

Proceso de impregnación a vacío: La EI fue preparada utilizando un homogeneizador Ultraturrax (UTL 50 INLINE Janke \& Kunkel IKA- Labortechnik), a 10.000rpm, durante $10 \mathrm{~min}$. Las UF fueron sometidas a IV aplicando una primera etapa de presión de vacío $(20,0 \mathrm{kPa})$, durante 5 min y restableciendo, posteriormente, la presión atmosférica (presión barométrica local $=85,3 \mathrm{kPa}$ ), manteniendo las muestras sumergidas, durante 5 min más (Peña et al. 2015). El cálculo de los parámetros de impregnación para uchuvas enteras, se llevó a cabo, determinándose la fracción volumétrica de impregnación ( $\mathrm{X}_{1}$ y X, $\mathrm{m}^{3} \mathrm{SI} / \mathrm{m}^{3} \mathrm{UF}$ ), deformación volumétrica $\left(\gamma_{1} \mathrm{y} \gamma, \mathrm{m}^{3} / \mathrm{m}^{3} \mathrm{UF}\right)$, al final de la etapa de vacío y al final del proceso, respectivamente, y la porosidad efectiva $\left(\varepsilon, \mathrm{m}^{3}\right.$ gas $/ \mathrm{m}^{3}$ UF). Para la El, se fijó una relación de adición de CFA, de acuerdo con el valor diario recomendado (VDR) de nutrientes, establecidos por la norma colombiana vigente, para niños mayores de 4 años y adultos (Ministerio de Protección Social, 2011), de manera que se obtuviera, aproximadamente, el 10\% VDR, para el Ca (100mg) y el 15\% VDR, para las vitamina $\mathrm{B}_{9}(60 \mu \mathrm{g}), \mathrm{C}(9 \mathrm{mg}), \mathrm{D}_{3}(1,5 \mu \mathrm{g})$ y E $(4,5 \mathrm{mg}$ DL- $\alpha$-tocoferol acetato), sobre la base de $100 \mathrm{~g}$ de UF.

Deshidratación por aire caliente: Para el proceso de deshidratación, se utilizó un secador de bandejas tipo túnel (Centricol S.A.), construida en acero inoxidable y conformada por un ventilador, un sistema de calentamiento por resistencia y una cámara de secado con 3 bandejas, en malla perforada, con un volumen de carga de $6,5 \times 10^{-4} \mathrm{~m}^{3} /$ bandeja, una balanza, anemómetros, termohigrómetros y un sistema integrado de adquisición de datos. El proceso se desarrolló a $60^{\circ} \mathrm{C}$ y $2 \mathrm{~m} / \mathrm{s}$, hasta que el producto alcanzó una $\mathrm{a}_{\mathrm{w}} \approx 0,600$; en un tiempo de proceso de 20,1 $\pm 1,1$ horas.

Almacenamiento: Las UISAC, se almacenaron a temperaturas controladas de 4,20 y $30^{\circ} \mathrm{C}$, en bolsas plásticas, con multicapa de poliamida-polietileno, marca ALICO S.A., con barrera al vapor de agua $\left(<15 \mathrm{~g} / \mathrm{m}^{2} / 24 \mathrm{~h} / \mathrm{atm}, \mathrm{T}=38^{\circ} \mathrm{C}\right), \mathrm{O}_{2}$ $\left(60 \mathrm{cc} / \mathrm{m}^{2} / 24 \mathrm{~h} / \mathrm{atm}, 23^{\circ} \mathrm{C}\right), \mathrm{N}_{2}$ y CO . El envasado, se realizó en condiciones atmosféricas (SV) y con aplicación de vacío (CV).

Determinación analítica de los CFA: El Ca, se determinó por absorción atómica con flama, basada en la Norma Téc- 
nica Colombiana 5151 de 2003. La extracción de las vitaminas, se realizó tomando alícuotas de, aproximadamente, $7 \mathrm{~g}$ de UISAC, obtenidos por homogeneización, en un equipo Ultraturrax (IKA ${ }^{\circledast}$ T25 digital), a 10.000 rpm, durante 5 min. La vitamina $B_{9}$, se extrajo, mediante la metodología propuesta por Arcot \& Shrestha (2005); la vitamina C, por el método de Gutiérrez et al. (2007) y las vitaminas liposolubles (Dз y E), por el método descrito por Cortés-Rodríguez et al. (2016a). La cuantificación de las vitaminas, se realizó por cromatografía líquida de alta resolución, en un equipo marca Shimadzu (serie 204 Prominence 20 $\AA$ ), provisto de un detector UV/VIS, con arreglo de diodos, una columna C18 RP- $5 \mu \mathrm{m}$ $4,0 \mathrm{~mm}$ x $250 \mathrm{~mm}$ y un flujo de $1,0 \mathrm{~mL} / \mathrm{min}$. Para las vitamina B9 y C, se evaluó el extracto acuoso, usando una fase móvil de ácido Orto-Fosfórico (85\%, 0,02M, pH =3,0), a una temperatura de horno de $35^{\circ} \mathrm{C}$, con una detección y tiempos de retención de 283nm, 244nm y 7,6 \pm 0,1; 4,5 \pm 0,1 min, respectivamente, mientras que para las vitaminas $D_{3}$ y $E$, se evaluó el extracto oleoso, con una fase móvil, acetonitrilo: metanol (25:75), una temperatura de horno de $45^{\circ} \mathrm{C}$ y una detección y tiempos de retención de $264 \mathrm{~nm}$, 285nm y $8,9 \pm$ 0,0 y $11,8 \pm 0,1 \mathrm{~min}$, respectivamente.

Propiedades fisicoquímicas y ópticas: Se determinó el contenido de humedad en base húmeda $\left(\mathrm{H}_{\mathrm{bh}}\right)$, por el método termo gravimétrico, norma AOAC 934.06 (AOAC, 1990); la $\mathrm{a}_{\mathrm{w}}$, con un higrómetro de punto de rocío, a $25^{\circ} \mathrm{C}$, con un equipo AquaLAB Decagon serie 3TE, norma AOAC 978.18 (AOAC, 1990); el contenido de sólidos solubles (SS), con un refractómetro Leica Auto ABBE (escala 0 - 32 Brix), norma AOAC 932.12 (AOAC, 1990); la acidez por titulación con $\mathrm{NaOH} 0,1 \mathrm{~N}$, utilizando como indicador fenolftaleína, norma AOAC 942.15 (AOAC, 1990) y el pH con un potenciómetro Schott CG840B, norma AOAC 981.12 (AOAC, 1990). El color, se determinó mediante un espectro fotocolorímetro (X-RITE, modelo SP64), utilizando el iluminante D65, con el observador de $10^{\circ}$, como referencia y ventana de observación de $4 \mathrm{~mm}$, obteniendo, a partir de los espectros de reflexión, las coordenadas de color del CIE-L*a*b, donde L* es un indicador de la luminosidad, cromaticidad a* verde (-) a rojo $(+)$ y cromaticidad b* azul (-) a amarillo $(+)$.

Diseño experimental y análisis de datos: Completamente aleatorizado con arreglo factorial, considerando tres factores: temperatura: 4,20 y $30^{\circ} \mathrm{C}$, tiempo: $0,1,2,3,4,5,6$ meses y envasado: CV, SV. Los resultados fueron analizados a partir de ANOVAS, utilizando el método LSD (mínimas diferencias significativas), como método de comparaciones múltiples, con un nivel de confianza del $95 \%(\alpha=0,05)$. Las variables dependientes, se evaluaron por triplicado, para cada condición de almacenamiento. Se utilizó el paquete estadístico STATGRAPHICS PLUS versión 5.1.

\section{RESULTADOS Y DISCUSIÓN}

Proceso de impregnación a vacío: La incorporación de la EI durante el proceso IV, se produce, principalmente, por el área del pedúnculo (punto de ruptura entre el fruto y el capacho) (Peña et al. 2013a); además, el fruto posee una película cérea de características semipermeables $(\approx 95 \%)$ (Cortés- Rodríguez et al. 2015), que influye negativamente en la respuesta a la IV, restringiendo la transferencia de masa de la EI durante el proceso.

Los resultados mostraron que la uchuva es una matriz alimentaria adecuada para el proceso de IV, permitiendo la incorporación de la EI en los espacios intercelulares, evidenciándose con la porosidad efectiva obtenida $\left(\varepsilon_{e}=6,6 \pm\right.$ $0,9 \%)$; sin embargo, en la etapa de vacío, se presenta salida de líquido nativo del interior de la estructura $\left(\mathrm{X}_{1}=-4,2 \pm\right.$ 1,1\%) (Cortés-Rodríguez et al. 2016a), lo que produce un ligero colapso volumétrico de expansión $\left(\gamma_{1}=0,5 \pm 0,5 \%\right)$ (Cortés-Rodríguez et al. 2015). El restablecimiento de la presión atmosférica permite la incorporación de la EI al interior de la estructura $(X=5,9 \pm 0,8 \%)$ y un colapso volumétrico de compresión $(\gamma=-2,2 \pm 1,0 \%)$, resultado del acoplamiento del mecanismo hidrodinámico y del fenómeno de relajación y deformación que experimenta la estructura, por los cambios de presión (Yang et al. 2017). Algunas investigaciones han reportado valores similares de los parámetros de impregnación en uchuva entera: Peña et al. (2015) (X: 3,5 $\pm 0,7 \%, X_{1}:-3,5 \pm 0,5 \%, \gamma:-4,2 \pm 0,5 \%, \gamma_{1}:-0,3 \pm 0,2 \%$, $\left.\varepsilon_{e}: 8,2 \pm 0,2 \%\right)$ y en uchuvas semiesférica: Cortés-Rodríguez et al. (2016a) (X: 24,9 $\pm 1,0 \%, X_{1}:-10,6 \pm 0,1 \%, \gamma: 11,8 \pm$ $0,0 \% ; \gamma_{1}: 0,9 \pm 0,0 \%$ y $\varepsilon$ e: $2,7 \pm 0,1 \%$ ), los cuales, han identificado que la respuesta a la IV depende de múltiples factores: formulación y viscosidad del líquido de impregnación, forma del fruto, condiciones del proceso (tiempos y presiones en cada etapa), entre otros.

En la tabla 1, se presentan los niveles alcanzados de los CFA incorporados por IV, sobre la base de $100 \mathrm{~g}$ de UF, teniendo en cuenta, dentro del balance de materia, que los contenidos iniciales de Ca y vitamina C en la UF fueron 1,6 \pm 3,6 y 19,8 $\pm 3,9 \mathrm{mg} / 100 \mathrm{~g}$ UF, respectivamente. Se observa que el proceso IV confiere un cambio composicional positivo causada por los cambios de presión, durante la acción del mecanismo hidrodinámico (Fito et al. 1996). Esta situación ha sido descrita en uchuva (Cortés-Rodríguez et al. 2016b; Peña et al. 2015) y en otras matrices alimentarias: manzana (Cortés-Rodríguez et al. 2016b; Castagnini et al. 2015) y plátano (Dávila et al. 2016), entre otros. 
Tabla 1. Contenidos de CFA en las UI sobre la base de $100 \mathrm{~g}$ de UF.

\begin{tabular}{|c|c|}
\hline CFA & Cantidad \\
\hline Calcio & $95,7 \pm 2,0 \mathrm{mg}$ \\
\hline Vitaminas $\mathrm{B}_{9}$ & $118,9 \pm 5,2 \mu \mathrm{g}$ \\
\hline Vitaminas C & $27,2 \pm 0,9 \mathrm{mg}$ \\
\hline Vitaminas $\mathrm{D}_{3}$ & $1,7 \pm 0,1 \mu \mathrm{g}$ \\
\hline Vitaminas $\mathrm{E}$ & $4,3 \pm 0,6 \mathrm{mg}$ \\
\hline
\end{tabular}

Estabilidad de los CFA durante el proceso de secado: El proceso de secado permitió obtener UISAC con valores de aw del orden de 0,593 $\pm 0,009$, considerado dentro del rango de seguridad microbiana (Stevenson et al. 2015). Bajo las condiciones utilizadas, se produjeron pérdidas de los CFA del orden de 2,7 $\pm 1,0 \% ; 32,8 \pm 4,1 \% ; 42,9 \pm 5,8 \% ; 1,1$ $\pm 0,3 \%$ y $3,4 \pm 2,2 \%$ para Ca y vitaminas $\mathrm{B}_{9}, \mathrm{C}, \mathrm{D}_{3}$ y E, respectivamente. Las bajas pérdidas presentadas por las vitaminas $\mathrm{D}_{3}$ y E, se podrían atribuir a la protección ofrecida por la matriz seca colapsada, al quedar los CFA encapsulados en su interior, además, por el carácter hidrofóbico de las formas químicas utilizadas (Betoret et al. 2015). Para el Ca, adicionalmente, su conservación se le atribuye a la fijación de este en la matriz por su interacción con los componentes pécticos de las paredes celulares (Gras et al. 2003) y su poca interacción con otros componentes (Peña et al. 2013a).

Las vitaminas hidrosolubles $\mathrm{B}_{9}$ y $\mathrm{C}$ representaron las mayores pérdidas, por estar directamente influenciada por la temperatura y el tiempo de secado (Cabrera-Ordoñez et al. 2017); además, puede estar presentándose una salida del líquido de impregnación posterior a la IV (Peña et al. 2015). Por otro lado, la vitamina $C$ podría experimentar una degradación enzimática, así, como reacciones de óxido reducción, que la convierten a ácido deshidroascórbico (Sánchez-Chávez et al. 2015). Algunas investigaciones han reportado el efecto del proceso de secado de la uchuva sobre los CFA: De Jesús-Jungueira et al. (2017) reportaron pérdidas de vitamina $\mathrm{C}$ en secado por convección, del $77,4 \%$, a $60^{\circ} \mathrm{C}$ y $2 \mathrm{~m} / \mathrm{s}$; Hernández-Sandoval et al. (2014) informaron de pérdidas de vitaminas $\mathrm{B}_{9}$ y $\mathrm{C}$ en polvo de uchuva, obtenida por secado por aspersión del 9,1 y 30,3\%, respectivamente; Aredo et al. (2012) obtuvieron pérdidas de vitamina C, del 74,3 y $68,8 \%$, en uchuva deshidratada con y sin pretratamiento con $\mathrm{NaCl}$ y, por otro lado, Cortés-Rodríguez et al. (2015), no hallaron pérdidas en $\mathrm{Ca}$ ni vitaminas $\mathrm{C}$ y $\mathrm{B} 9$ en uchuva liofilizada.

En este contexto, las UISAC a la salida del proceso o inicio del almacenamiento alcanzaron niveles de $472,7 \pm 49,7 \mathrm{mg}$, $243,6 \pm 35,6 \mu \mathrm{g}, 34,7 \pm 9,9 \mathrm{mg}, 5,9 \pm 1,5 \mu \mathrm{g} \mathrm{y} 14,7 \pm$ 3,9mg, para Ca y vitaminas $\mathrm{B}_{9}, \mathrm{C}, \mathrm{D}_{3}$ y $\mathrm{E}$, respectivamente, para una porción de $100 \mathrm{~g}$ UISAC.
Estabilidad de los CFA durante el almacenamiento: La tabla 2 presenta los valores medios más la desviación estándar de los CFA, en una porción de $100 \mathrm{~g}$ de UISAC, durante el almacenamiento. El ANOVA no mostró diferencias estadísticas ( $p>0,05)$ en los CFA, por efecto del factor empacado, lo cual, se podría deber al buen nivel de protección que ofrece la matriz seca de la uchuva frente a la oxidación del compuesto, dificultando la difusión de $\mathrm{O}_{2}$ hasta las moléculas de los CFA. Por otro lado, sí hubo diferencias estadísticas $(p<0,05)$ en las Vitaminas B, $C$, D 3 y E, por efecto del factor tiempo y en las vitaminas $C$, y $D_{3}$, por efecto de la temperatura.

El Ca no estuvo afectado por ninguna variable independiente, lo que revalida la buena estabilidad, mencionada anteriormente, en el proceso de secado (Peña et al. 2013b; Gras et al. 2003), por lo que el promedio global, durante los 6 meses de almacenamiento, fue de 445,5 $\pm 61,7 \mathrm{mg} / 100 \mathrm{~g}$ UISAC.

La vitamina B9 presentó un comportamiento similar hasta el mes 2, posteriormente, se observa una tendencia a disminuir con el tiempo, siendo mayor la disminución con el incremento de la temperatura y alcanzando en el mes 6 , pérdidas del $29,0,46,9$ y $49,9 \%$ a 4,20 y $30^{\circ} \mathrm{C}$, respectivamente. Esta degradación podría ser atribuida a la integración de diversos factores: efecto térmico, luz, permeabilidad $\mathrm{al}_{2}$ en el empaque y a los cambios de pH (Olivares et al. 2006).

Hernández-Sandoval et al. (2014) registraron un efecto de la temperatura y el tiempo sobre la vitamina $\mathrm{B}_{9}$ en uchuva en polvo, empacado en condiciones atmosféricas, durante 6 meses, a 4,20 y $30^{\circ} \mathrm{C}$, siendo las pérdidas de 2,6, 6,0 y $13,8 \%$, respectivamente. Peña et al. (2013b) reportaron en uchuva, mínimamente procesada, adicionada con CFA, pérdidas de vitamina $\mathrm{B}_{9}$, del $27,5 \%$, durante 21 días, a $4^{\circ} \mathrm{C}$. Strålsjö et al. (2003) encontraron una parcial estabilidad del escaramujo o rosa canina, por la presencia de agentes reductores, como la vitamina $\mathrm{C}$, que ejerce un efecto protector desde el proceso de secado. Por otro lado, Hernández et al. (2008) relacionan la pérdida de la vitamina $\mathrm{B}_{9}$ en la fresa, con la concentración de $\mathrm{O}_{2}$.

La vitamina $C$ presentó degradación con el tiempo y con el incremento de la temperatura, alcanzando pérdidas del 15,3, 34,3 y $42,3 \%$, para 4,20 y $30^{\circ} \mathrm{C}$, respectivamente. Diversos factores afectan la estabilidad oxidativa de esta la vitamina $\mathrm{C}$ : temperatura, $\mathrm{O}_{2}$, luz, metales y la presencia de ácido ascórbico oxidasa (Gębczyński et al. 2017).

La mejor estabilidad de la vitamina $\mathrm{C}$ fue a $4^{\circ} \mathrm{C}$, lo que se podría asociar a un efecto sinérgico por la presencia de otras sustancias, como ácidos orgánicos, azúcares reductores propios de la fruta y producidos por hidrólisis de polifenoles. 
Tabla 2. Contenido de CFA en una porción de $100 \mathrm{~g}$ de UISAC, durante el almacenamiento.

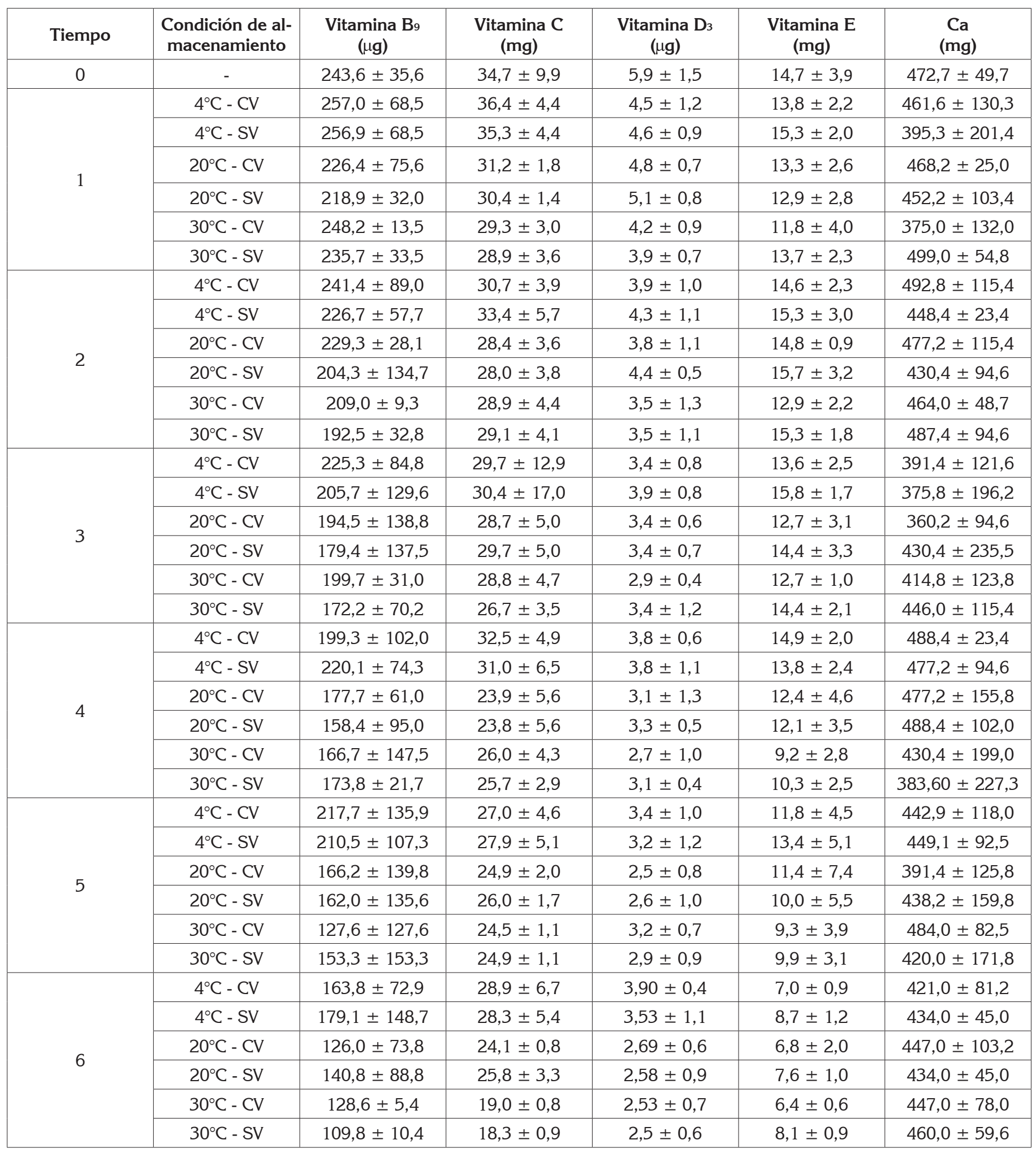


Por otro lado, el pH en la UISAC $(3,5 \pm 0,1)$ juega un papel importante en su estabilidad, ya que la vitamina podría estar completa o parcialmente protonada, lo cual, representa un estado de mayor estabilidad (Gębczyński et al. 2017). Hernández-Sandoval et al. (2014) reportaron pérdidas de vitamina $\mathrm{C}$ en uchuva en polvo, empacada en condiciones atmosféricas, durante 6 meses, a 4, 20 y $30^{\circ} \mathrm{C}$, de 30,0, 40,6 y $71,8 \%$, respectivamente y empacada al vacío, de 29,6, 37,6 y $68,3 \%$, respectivamente. De Jesús-Junqueira et al. (2017) informaron pérdidas entre 54 y 77\%, luego del secado por convección, a $60^{\circ} \mathrm{C}$ y a $2 \mathrm{~m} / \mathrm{s}$. Mendoza-Corvis et al. (2017) hallaron una pérdida del $43,6 \%$ de vitamina $C$, en un producto en polvo de mango y lactosuero, durante 8 semanas, mientras que a $4^{\circ} \mathrm{C}$, se conservó.

La vitamina $\mathrm{D}_{3}$ presenta, en los primeros 3 meses, una pérdida gradual con el tiempo de almacenamiento y con el incremento de temperatura; posteriormente, la vitamina se comporta de manera muy estable, a $4^{\circ} \mathrm{C}$, mientras que a 20 y $30^{\circ} \mathrm{C}$, continúa su degradación, con una pendiente menor. Las pérdidas durante los 6 meses fueron del 30,1, 56,3 y $60,0 \%$ a 4,20 y $30^{\circ} \mathrm{C}$, respectivamente. Se considera que esta vitamina es estable en ausencia de agua y, a bajas temperaturas, no le afecta la presencia del $\mathrm{O}_{2}$, por lo que puede ser empacada en condiciones atmosférica y es menos susceptible a pérdidas por oxidación, que las vitaminas $\mathrm{E}$, $\beta$-caroteno y retinol (Sánchez-Chávez et al. 2015); no obstante, a condiciones ácidas, como el caso de la UISAC, se puede isomerizar, siendo la oxidación la ruta predominante para su descomposición; además, se ha demostrado que la exposición a la luz puede ocasionar pérdidas significativas de esta vitamina (Peña et al. 2015).

Pocos autores reportan la estabilidad de vitamina D en frutas frescas o deshidratadas: Peña et al. (2013b) encontraron una conservación de la vitamina $\mathrm{D}_{3}$ en uchuva, mínimamente procesada, durante 21 días, a $4^{\circ} \mathrm{C}$, mientras que Ruiz et al. (2017) señalan pérdidas del orden de $50-47 \%$ de vitamina $\mathrm{D}_{3}$, en polvo de aguacate, almacenado a $35^{\circ} \mathrm{C}$, durante 6 meses y empacado al vacío y $\mathrm{N}_{2}$.

La vitamina $\mathrm{E}$, en forma de acetato, presentó una buena estabilidad durante los primeros 3 meses, favorecida por las condiciones ácida del medio; a partir de este tiempo, se potencia el proceso degradativo, llegando las pérdidas a valores de 49,4\%, a los 6 meses. La degradación del DL- $\alpha$-tocoferol acetato, se podría deber a diversos factores, que incluyen las reacciones oxidativas, la luz, la presencia de diferentes metales que catalizan las reacciones oxidativas y, finalmente, por los radicales libres presentes (Hernández et al. 2008).

Peña et al. (2013b) encontraron en uchuva, mínimamente procesada, pérdidas de vitamina $C$, del $9,4 \%$, durante 21 días de almacenamiento, a $4^{\circ} \mathrm{C}$. Cortés et al. (2009) repor- taron en manzana, adicionada con vitamina E por IV y liofilizada, durante 180 días, pérdidas de 33,6, 48,5 y 47,0\% a 4,20 y $30^{\circ} \mathrm{C}$, respectivamente, mientras que secada por convección, a $40^{\circ} \mathrm{C}$, las pérdidas fueron de $6,9,27,7$ y 37,5 a 4,20 y $30^{\circ} \mathrm{C}$, respectivamente (Cortés et al. 2007). En fresa, mínimamente procesada adicionada, por IV con vitamina E, no se reportaron pérdidas durante el almacenamiento, a $4^{\circ} \mathrm{C}$ (Restrepo et al. 2009).

Propiedades fisicoquímicas y ópticas: La figura 1 presenta los valores medios con los intervalos LSD (95\%) de los parámetros fisicoquímicos de las UISAC, durante 6 meses de almacenamiento. El ANOVA presentó diferencias significativas $(p<0,05)$ en la $a_{w}$ y $H_{b h}$ con respecto a los factores tipo de empacado, temperatura y tiempo y, los SS, respecto a la temperatura, mientras que el $\mathrm{pH}$ y la acidez no presentaron diferencias significativas ( $p>0,05)$, siendo sus valores globales $3,5 \pm 0,1$ y $2,1 \pm 0,3 \%$, respectivamente.

La evolución en el tiempo de la aw y Hbh es muy similar para cada temperatura, al estar ambas relacionadas, a través de las curvas de sorción. Se observa que las muestras almacenadas a $4^{\circ} \mathrm{C}$ tienen un comportamiento prácticamente estable durante el período de almacenamiento, siendo la variación entre 0,580-0,620 ( $\mathrm{aw}$ ) y de $25-29 \%(\mathrm{Hbh})$, mientras que, a 20 y $30^{\circ} \mathrm{C}$, se produce una disminución a lo largo del tiempo, siendo más acentuada a $30^{\circ} \mathrm{C}$; este descenso, se puede deber a diferencias en el potencial químico del agua entre el ambiente interno del empaque y el producto. La dependencia a la temperatura, se debe, probablemente, al incremento de la presión de vapor del agua, a medida que aumenta la temperatura y de los coeficientes de transferencia de masa, con lo que se va produciendo una pérdida mayor de agua de la muestra, a temperaturas más altas (Yao et al. 2015). Las UISAC, a los 6 meses de almacenamiento y a las condiciones de aw obtenidas $(0,530-0,600)$, pueden ser catalogadas como alimentos de humedad intermedia ( $\mathrm{H}_{\mathrm{bh}} \approx 22-30 \%$ ); bajo estas condiciones existe una inhibición del crecimiento de patógenos, hongos y levaduras (Stevenson et al. 2015).

Los SS presentaron un comportamiento similar durante los primeros 5 meses, pero con alta variabilidad (56-67\%), lo cual, se podría deber a varios factores: a las diferencias en los niveles de maduración de la UF, a las diferencias en los niveles de IV de las muestras a la entrada del proceso de secado y a una posible hidrólisis del almidón y de los polisacáridos de la pared celular, que dan origen a azúcares solubles (Balaguera-López et al. 2014). En el mes 6, se observa una tendencia a incrementar los SS y mucho más con el incremento de la temperatura, lo que se podría explicar por la reducción de la Hbh.

Algunos autores señalan comportamientos diversos de las propiedades fisicoquímicas de la uchuva. Peña et al. (2013b), 

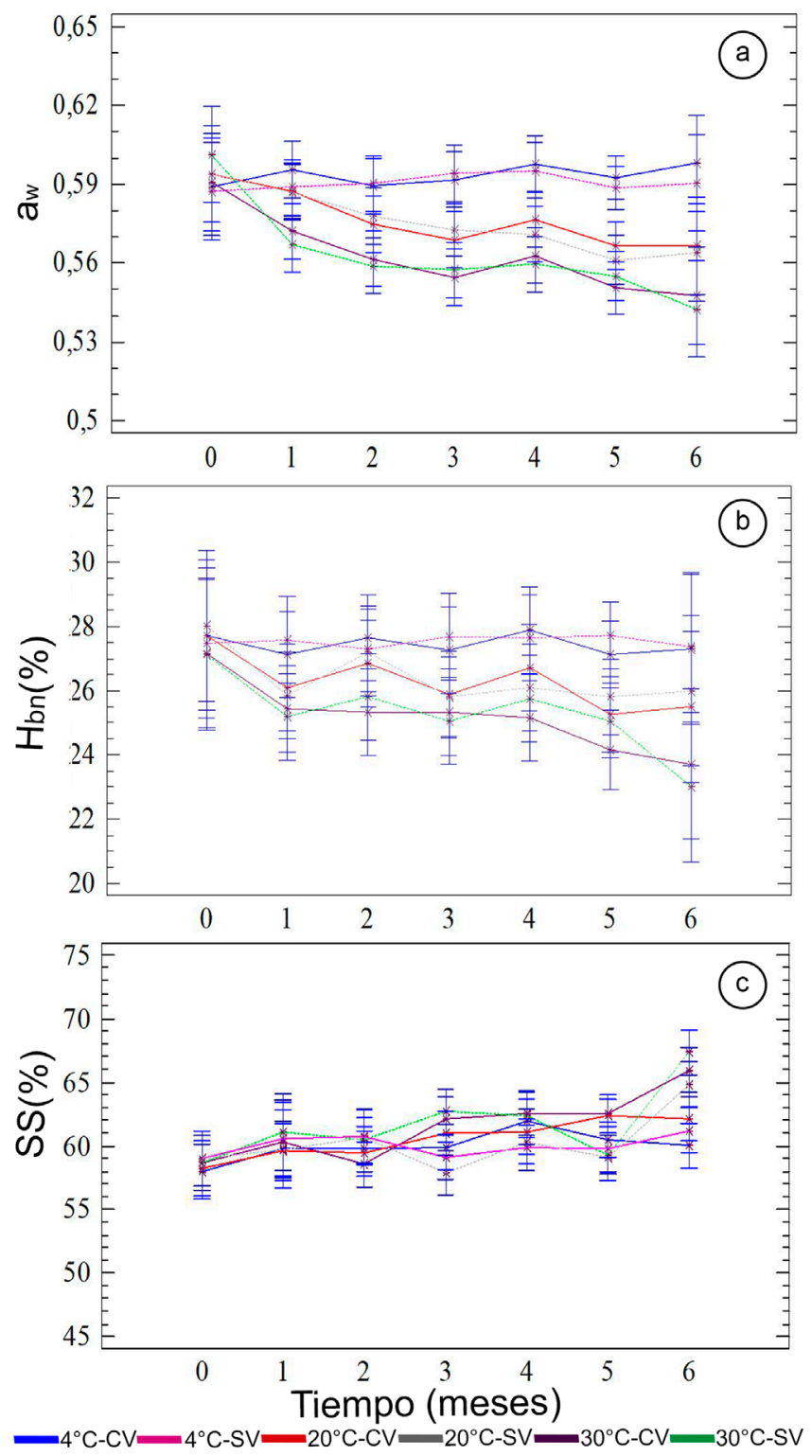

Figura 1. Evolución de las propiedades a. aw, b. Hbh y c. SS en las UISAC, durante el almacenamiento.

en uchuva impregnada con CFA, mostraron ligeras fluctuaciones del $\mathrm{pH}(3,5-3,7)$, acidez $(1,4-1,8 \%)$, aw $(0,980$ $0,984)$ y SS $(14,6-15,8 \%)$, durante 21 de almacenamiento. Reyes et al. (2017), en UF adicionada con Ca, cambios en el pH $(3,4-3,6)$ y conservación de los SS $(\approx 16,3 \%)$, durante 35 días, a $14^{\circ} \mathrm{C}$ y Balaguera-López et al. (2014) encontraron un comportamiento similar de los SS en uchuvas, con y sin cáliz, durante 22 días de almacenamiento, a $16^{\circ} \mathrm{C}$.

La figura 2 presenta los valores medios con los intervalos LSD $(95 \%)$ de los parámetros de color (L*, a* y b*) de las UISAC, durante los 6 meses de almacenamiento. El ANOVA presentó diferencias significativas $(p<0,05)$, en los parámetros de color, con respecto a los factores temperatura y tiempo, mientras que el tipo de empacado (SV, CV), no fue relevante a las mismas condiciones de almacenamiento. A $4^{\circ} \mathrm{C}$, las UISAC mostraron un comportamiento uniforme del color durante los 180 días, mientras que al aumentar la temperatura almacenamiento $\left(20\right.$ y $\left.30^{\circ} \mathrm{C}\right)$, disminuyen con el incremento del tiempo, siendo más incidente a mayor temperatura. Esta situación hace que las muestras se perciban más oscuras $\left(<L^{*}\right)$, lo cual, se podría asociar a reacciones de pardeamiento no enzimático, por la presencia de azúcares reductores (glucosa) y aminoácidos presentes en la proteína aislada de soja (lisina y arginina); además, por otras reacciones de carácter oxidativo, asociadas al ácido ascórbico presente, que contribuyen igualmente al oscurecimiento (Mendoza-Corvis et al. 2015). La disminución de los parámetros 
$a^{*}$ y b* a 20 y $30^{\circ} \mathrm{C}$ identifica una pérdida de las tonalidades rojas y amarillas, lo cual, se podría atribuir a la degradación de pigmentos carotenoides, propios del fruto (Mendoza-Corvis et al. 2015). La evolución del color en el plano cromático a*b*, durante los 6 meses, desplaza las muestras desde la zona de los anaranjados hacia la zona de los grises, lo cual, es correspondiente a una menor intensidad o saturación de color que, sumado a la disminución de L*, hace percibir las muestras como opacas y pardas.
El proceso de IV permite la incorporación efectiva de CFA en la matriz de uchuva. El proceso de secado por aire por convección, a $60^{\circ} \mathrm{C}$ y $2 \mathrm{~m} / \mathrm{s}$, permitió obtener productos de UISAC seguros microbiológicamente; no obstante, evidenció unas pérdidas importantes, principalmente, en las vitaminas B 9 y C. Durante los 6 meses de almacenamiento, el Ca se conserva en la matriz, mientras que las vitaminas $\mathrm{B}_{9}, \mathrm{C}, \mathrm{D}_{3}$ y E están afectadas por el tiempo o la temperatura. Se es-
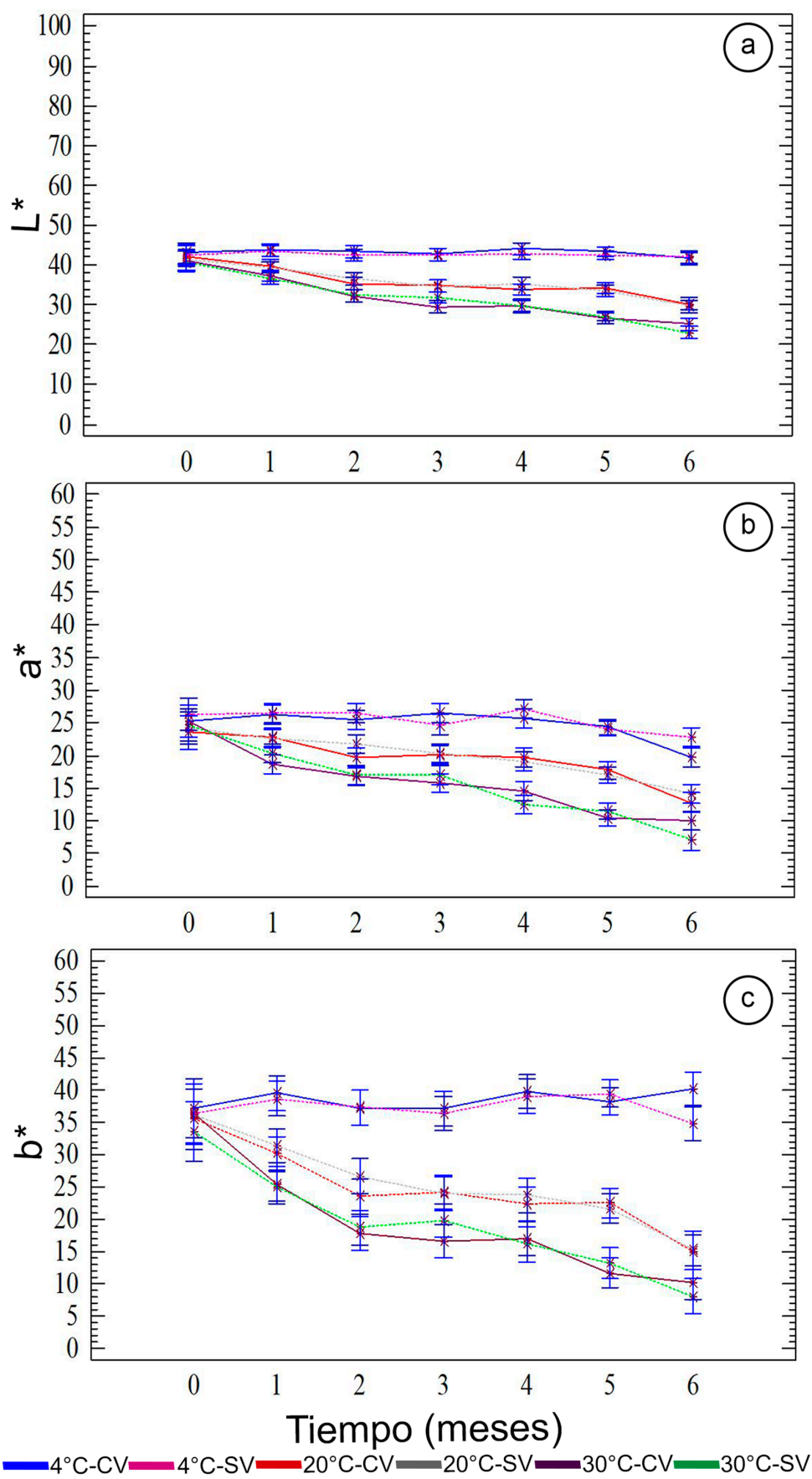

Figura 2. Evolución de Los parámetros de color a. L*, b. a* y c. b* en las UISAC, durante el almacenamiento. 
tableció que la mejor condición de almacenamiento de las UISAC fue a $4^{\circ} \mathrm{C}$ y empacado atmosférico, presentando los mayores contenidos de CFA y mejores atributos de calidad (fisicoquímicos y físicos). En el contexto de la normativa colombiana (Ministerio de Protección Social, 2011) y en concordancia con los descriptores permitidos en el etiquetado nutricional, $100 \mathrm{~g}$ de UISAC, almacenados durante 6 meses, a $4^{\mathrm{a}} \mathrm{C}, 20^{\circ} \mathrm{C}$ y $30^{\circ} \mathrm{C}$, aportan contenidos superiores al $20 \%$ del VDR, para cada nutriente, por lo que se permite declarar, como "Excelente fuente de Ca y vitaminas $\mathrm{B}_{9}, \mathrm{C}, \mathrm{D}_{3}$ y E".

Conflicto de intereses: El manuscrito del presente trabajo fue preparado y revisado con la participación de todos los autores, quienes declaramos que no existe conflicto de intereses que ponga en riesgo la validez de los resultados presentados.

\section{REFERENCIAS}

1. ÁlVAREZ-HERRERA, J.; FISCHER, G.; VÉLEZSANCHEZ, J.E. 2015. Production of cape gooseberry (Physalis peruviana L.) fruits with different irrigation coefficients and frequencies and doses of calcium. Rev. Col. Ciencias Hort. 9(2):222-233. http://dx.doi. org/10.17584/rcch.2015v9i2.4177

2. AOAC. 1990. Official methods of analysis of AOAC international. Vol. 2. $15^{\text {th }}$ Ed. Methods 934.06, 978.18, 932.12, 942.15, 981.12. Association of official analytical chemists. Arlington, Virginia (USA). 1298p.

3. ARCOT, J.; SHRESTHA, A. 2005. Folate: methods of analysis. Trends Foods Sci. Tecnol. (UK). 16(6-7):253266. https://doi.org/10.1016/j.tifs.2005.03.013

4. AREDO, V.; ARTEAGA, A.; BENITES, B.; GERÓNIMO, G. 2012. Comparación entre el secado convectivo y osmoconvectivo en la pérdida de vitamina $C$ de Aguaymanto (Physalis peruviana) con y sin pre-tratamiento de $\mathrm{NaOH}$. Agroind. Sci. (Perú). 2:126-131.

5. BALAGUERA-LÓPEZ, H.E.; MARTÍNEZ, C.A.; HERRERA-ARÉVALO, A. 2014. Papel del cáliz en el comportamiento poscosecha de frutos de uchuva (Physalis peruviana L.) ecotipo Colombia. Rev. Col. Ciencias Hort. 8(2):181-191.

6. BETORET, E.; BETORET, N.; ROCCULI, P.; DALLAROSA, M. 2015. Strategies to improve food functionality: Structure-property relationships on high pressures homogenization, vacuum impregnation and drying technologies. Trends Foods Sci. Tecnol. 46(1):1-12. https://doi.org/10.1016/j.tifs.2015.07.006
7. BIGLIARDI, B.; GALATI, F. 2013. Innovation trends in the food industry: The case of functional foods. Trends Foods Sci. Tecnol. 31(2):118-129. https://doi. org/10.1016/j.tifs.2013.03.006

8. CABRERA-ORDOÑEZ, Y.A.; ESTRADA, E.M.; CORTÉSRODRÍGUEZ, M. 2017. The influence of drying on cape gooseberry (Physalis peruviana L.) fruits added with active components. Acta Agron. (Colombia). 66(4):512-518. https://doi.org/10.15446/acag. v66n4.59507

9. CARCIOFI, B.; TELEKEN, J.; BERTELLI, V.; PRAT, M.; LAURINDO, J. 2015. Experimental approach to evaluate the influence of characteristic length on the dynamics of biphasic flow in vacuum impregnation. Chem. Eng. Sci. (Australia). 137:875-883. https://doi. org/10.1016/j.ces.2015.07.052

10. CARVALHO, C.P.; VILLAÑO, D.; MORENO, D.; SERRANO, M.; VALERO, D. 2015. Alginate edible coating and cold storage for improving the physicochemical quality of cape gooseberry (Physalis peruviana L.). J. Food Sci. Nutr. (USA). 1(1):1-7. http://doi.org/10.24966/ FSN-1076/100002

11. CASTAGNINI, J.; BETORET, N.; BETORET, E.; FITO, P. 2015. Vacuum impregnation and air drying temperature effect on individual anthocyanins and antiradical capacity of blueberry juice included into an apple matrix. LWT Food Sci. Technol. (Swiss). 64(2):12891296. https://doi.org/10.1016/j.lwt.2015.06.044

12. CORTÉS-RODRÍGUEZ, M.; HERRERA-HERRERA, E.; GIL-GONZÁLEZ, J. 2016a. Semi-spherical shape of cape gooseberry (Physalis peruviana L.) impregnated with a fortificated emulsion. Biotecn. Sector Agropec. y Agroindust. (Colombia). 14(1):27-36. http://dx.doi. org/10.18684/BSAA(14)27-36

13. CORTÉS, M.; CHIRALT, A.; ARANGO, J.C. 2016b. Quality of dried apple products added with active compounds. Rev. Ciencias Agrícolas (Colombia). 33(2):55-61. http://dx.doi.org/10.22267/rcia.163302.52

14. CORTÉS-RODRÍGUEZ, M.; HERRERA, E.; RODRÍGUEZ, E. 2015. Experimental optimization of the freeze dry process of cape gooseberry added with active compounds by vacuum impregnation. Vitae. 22(1):47-56. http://dx.doi.org/10.17533/udea.vitae.v22n1a06

15. CORTÉS, M.; CHIRALT, A.; SUÁREZ, H. 2009. Influence of storage conditions on freeze-dried apple fortified with vitamin E. Vitae (Colombia). 16(1):31-41. 
16. CORTÉS, M.; OSÓRIO, A.; GARCÍA E. 2007. Manzana deshidratada fortificada con vitamina E utilizando la Ingeniería de Matrices. Vitae. 14(2):17-26.

17. DÁVILA, R.M.; CORTÉS, M.; GIL, J.H. 2016. Cambios físicos y fisicoquímicos durante el almacenamiento en plátano impregnado al vacío con soluciones antioxidantes. Biotecn. Sector Agropec. y Agroindust. 14(2):125-134. http://doi.org/10.18684/ BSAA(14)125-134

18. DE JESÚS-JUNQUEIRA, J.R.; GOMES-CORREA, J.L.; DE OLIVEIRA, H.M.; SOARES-AVELAR, R.I.; SALLESPIO, L.A. 2017. Convective drying of cape gooseberry fruits: Effect of pretreatments on kinetics and quality parameters. LWT - Food Sci. Technol. 82:404-410. https://doi.org/10.1016/j.lwt.2017.04.072

19. FITO, P.; ANDRÉS, A.; CHIRALT, A.; PARDO, P. 1996. Coupling of hydrodynamic mechanism and deformation-relaxion phenomena during vacuum treatments in solid porous food-liquid systems. J. Food Eng. (England). 27:229-240.

20. GĘBCZYŃSKI, P.; SKOCZEŃ-SŁUPSKA, R.; KUR, K. 2017. Effect of storage on the content of selected antioxidants and quality attributes in convection and freeze-dried pears (Pyrus communis L.). Ital. J. Food Sci. 29:454-462.

21. GRAS, M.L.; VIDAL, D.; BETORET, N.; CHIRALT, A.; FITO, P. 2003. Calcium fortification of vegetables by vacuum impregnation: Interactions with cellular matrix. J. Food Eng. 56(2-3):279-284. https://doi. org/10.1016/S0260-8774(02)00269-8

22. GUTIÉRREZ, T.; HOYOS, O.; PÁEZ, M. 2007. Determinación del contenido de ácido ascórbico en uchuva (Physalis peruviana L.), por cromatografía líquida de alta resolución (HPLC). Biotecn. Sector Agropec. y Agroindust. 5(1):70-79.

23. HERNÁNDEZ, P.; ALMENAR, E.; DEL VALLE, V.; VÉLEZ, D.; GAVARA, R. 2008. Effect of chitosan coating combined with postharvest calcium treatment on strawberry (Fragaria ananassa) quality during refrigerated storage. Food Chem. (UK). 110(2):428-435. https:// doi.org/10.1016/j.foodchem.2008.02.020

24. HERNÁNDEZ-SANDOVAL, G.R.; CORTÉS-RODRÍGUEZ, M.; CIRO-VELAZQUEZ, H.J. 2014. Effect of storage conditions on quality of a functional powder of cape gooseberry obtained by spray drying. Rev. U.D.C.A Act. \& Div. Cient. (Colombia). 17(1):139-149.
25. MENDOZA-CORVIS, F.; HERNÁNDEZ, E.; RUIZ, L. 2015. Effect of blanching on the color and thermal degradation kinetics of vitamin c of mango of hilacha pulp (Mangifera indica var Magdalena river). Información Tecnológica (Chile). 3:9-16. https://doi.org/10.4067/ S0718-07642015000300003

26. MENDOZA-CORVIS, F.; ARTEAGA-MÁRQUEZ, M.R.; PÉREZ-SILVA, O.A. 2017. Degradación de la vitamina $\mathrm{C}$ en un producto de mango (Mangifera indica L.) y lactosuero. Corpoica Cienc. Tecnol. Agropecuaria (Colombia). 18(1):125-137. https://doi.org/10.21930/ rcta.vol18_num1_art:563

27. MINISTERIO DE PROTECCIÓN SOCIAL. 2011. Resolución 333 del 10 de febrero de 2011. Por la cual se establece el reglamento técnico sobre los requisitos de rotulado o etiquetado nutricional que deben cumplir los alimentos envasados para consumo humano. (Colombia).

28. NORMA TÉCNICA COLOMBIANA -NTC- 4580. ICONTEC (Colombia). 1999. Frutas frescas. Uchuva. Especificaciones. Primera actualización, Bogotá, Colombia. 15p.

29. NORMA TÉCNICA COLOMBIANA -NTC 5151. ICONTEC (Colombia). 2003. Alimentos para animales. Determinación de los contenidos de calcio, cobre, hierro, magnesio, manganeso, potasio, sodio y zinc. Método usando espectrometría de absorción atómica, Bogotá, Colombia. 17p.

30. OLIVARES, A.B.; BERNAL, M.J.; ROS, G.; MARTÍNEZ, C.; PERIAGO, M.J. 2006. Calidad de los datos del contenido en ácido fólico en vegetales recogidos en varias tablas de composición de alimentos españolas, y nuevos datos sobre su contenido en folatos. Nutr. Hosp. (España). 21(1):97-108.

31. OLIVARES-TENORIO, M.; DEKKER, M.; VAN BOEKEL, M.; VERKERK, R. 2017. Evaluating the effect of storage conditions on the shelf life of cape gooseberry (Physalis peruviana L.). LWT Food Sci. Technol. 80:523-530. https://doi.org/10.1016/j.lwt.2017.03.027

32. PEÑA, F.; CORTÉS-RODRÍGUEZ, M.; GIL-GONZÁLEZ, J. 2015. Minimally processed cape gooseberry vacuum impregnated with calcium and vitamins $\mathrm{B}_{9}, \mathrm{D}$ and $\mathrm{E}$. Biotecn. en el Sector Agropecuario y Agroindust. 13(1):110-119.

33. PEÑA, R.F.; CORTÉS, M.; MONTOYA, O.I. 2013a. Evaluation of the physicochemical, physical and 
sensory properties of fresh cape gooseberry and vacuum impregnated with physiologically active components. Vitae. 20(1):13-22.

34. PEÑA, R.F.; CORTÉS, M.; GIL, J.H. 2013b. Estabilidad Fisicoquímica y funcional de uchuva (Physalis peruviana L.) impregnada al vacío con calcio y vitaminas $\mathrm{B}_{9}$, D y E, durante el almacenamiento. Rev. Fac. Nal. Agr. Medellín (Colombia). 66(1):6929-6938.

35. RABIE, M.; SOLIMAN, A.; DIACONEASA, Z.; CONSTANTIN, B. 2015. Effect of pasteurization and shelf life on the physicochemical properties of physalis (Physalis peruviana L.) juice. J. Food Process. Preserv. (UK). 39(6):1051-1060. https://doi.org/10.1111/jfpp.12320

36. RESTREPO, A.M.; CORTÉS, M.; ROJANO, B. 2009. Determinación de la vida útil de fresa (Fragaria ananassa Duch.) fortificada con vitamina E. DYNA. (Colombia). 159(76):163-175.

37. REYES-MEDINA, A.J.; PINZÓN, E.H.; ÁLVAREZHERRERA, J.G. 2017. Effect of calcium chloride and refrigeration on the quality and organoleptic characteristics of cape gooseberry (Physalis peruviana L.). Acta. Agron. 66(1):15-20. http://dx.doi. org/10.15446/acag.v66n1.50610Acta

38. RUIZ RUIZ, M.P.; CORTÉS-RODRÍGUEZ, M.; GIL, J.H. 2017. Estabilidad del polvo de aguacate adicionado con componentes activos durante el almacenamiento. Biotecn. Sector Agropec. y Agroindust. 15(2):42-51. http://dx.doi.org/10.18684/ bsaa(v15)EdiciónEspecialn2.577

39. SÁNCHEZ-CHÁVEZ, W.; CORTEZ-ARREDONDO, J.; SOLANO-CORNEJO, M.; VIDAURRE-RUIZ, J. 2015. Cinética de degradación térmica de betacianinas, betaxantinas y vitamina $C$ en una bebida a base de jugo de remolacha (Beta vulgaris L.) y miel de abeja. Scientia Agropecuaria (Perú). 6(2):111-118.

40. STEVENSON, A.; CRAY, J.; WILLIAMS, J.; SANTOS, R.; SAHAY, R.; NEUENKIRCHEN, N.; MCCLURE, C.; GRANT, I.; HOUGHTON, J.; QUINN, J.; TIMSON,
D.; PATIL, S.; SINGHAL, R.; ANTÓN, J.; DIJKSTERHUIS, J.; HOCKING, A.; LIEVENS, B.; RANGEL, D.; VOYTEK, M.; GUNDE-CIMERMAN, N.; OREN, A.; TIMMIS, K.; MCGENITY, T.; HALLSWORTH, J. 2015. Is there a common water-activity limit for the three domains of life? ISME J. (Netherlands). 9(6):1333-1351. http://doi.org/10.1038/ismej.2014.219

41. STRÅLSJÖ, L.; ALKLINT, C.; OLSSON, M.; SJÖHOLM, I. 2003. Total folate content and retention in rosehips (Rosa ssp.) after drying. J. Agric. Food. Chem. (USA). 51(15):4291-4295. http://doi.org/10.1021/jf034208q

42. TAPPI, S.; TYLEWICZ, U.; ROMANI, S.; SIROLI, L.; PATRIGNANI, F.; DALLA ROSA, M.; ROCCULI, P. 2016. Optimization of vacuum impregnation with calcium lactate of minimally processed melon and shelflife study in real storage conditions. J. Food Sci. (USA). 81(11):E2734-E2742. https://doi.org/10.1111/17503841.13513

43. WORLD HEALTH ORGANIZATION -WHO-. 2003. Diet, nutrition, and the prevention of chronic diseases: report of a joint WHO/FAO expert consultation. (Suiza): WHO Library Cataloguing in Publication Data. 160p. Disponible desde Internet en: http://apps.who.int/iris/ bitstream/10665/42665/1/WHO_TRS_916.pdf__(con acceso el 17/0972017).

44. YANG, H.; WU, Q.; NG, L.; WANG, F. 2017. Effects of vacuum impregnation with calcium lactate and pectin methylesterase on quality attributes and chelate-soluble pectin morphology of fresh-cut papayas. Food Bioprocess Technol. (USA). 10:901-913. http://doi. org/10.1007/s11947-017-1874-7

45. YAO, C.; DONG, Z.; ZHAO, Y.; CHEN, G. 2015. Gas-liquid flow and mass transfer in a microchannel under elevated pressures. Chem. Eng. Sci. (Australia). 123:137145. https://doi.org/10.1016/j.ces.2014.11.005

Recibido: Enero 30 de2018

Aceptado: Octubre 14 de 2018

Cómo citar:

Cortés-Rodríguez, M.; Cabrera Ordoñez, Y.A.; Ortega-Toro, R. 2018. Efecto del almacenamiento sobre uchuva adicionada con componentes fisiológicamente activos y deshidratada por aire caliente. Rev. U.D.C.A Act. \& Div. Cient. 21(2):427-437. https://doi.org/10.31910/rudca.v21.n2.2018.989 